\title{
Equations for All the Primes Numbers
}

\author{
Andri Lopez \\ Department of Mathematics, Shool of Minas, Leon, Spain \\ ${ }^{*}$ Corresponding Author: andri3lopez@gmail.com
}

Copyright (C2013 Horizon Research Publishing All rights reserved.

\begin{abstract}
with this work the equation is defined for the primes numbers; this one work is based on additive
\end{abstract} theory number and arithmetical progressiones.

Keywords Arithmetic, Proposition, Equation 5, Equation 6, Equation Twin Prime Number

\section{Introduction}

In this work the equations are definite and demonstrated for all the prime numbers. I have to say that all my investigation was centred exclusively in the additive theory [1] and, arithmetic progression [2].

\section{Methodology}

The investigation has been exhaustive and extended in the time since, the aim, always it was to define an equation for the odd numbers of such form that his possible fectoring, was with the minor possible number of numbers. Obtained aim.

For definition a prime number is any entire number that takes the unit as dividing only ones the same number.

$$
\begin{gathered}
p \in(2 c+1) \\
\exists p \in(2 c)
\end{gathered}
$$

[1] We know that the process for generates odd numbers in sum is $(1+2+2+2+\ldots$.$) , consequently this is$ the same thing that $(3+2+2+2+\ldots$.$) is to say (3+2 n)$. Of general form we will say $(x+x n=2 c+1)$.

The minor odd number that generates other odd in $x(1+n)$ is $(x=3)$, in order that result is always an odd number, (n) has to (2b); with which it stay.

$$
3+2 b=2 c+1
$$

If now in this equality we want that all the odd numbers are multiples of three we will have.

$$
3+3 \cdot 2 b=3+6 b
$$

And, for other odd not multiple of three, simply we will add an even number, that is to say.

$$
\begin{aligned}
& 3+2 \cdot 3 b+2=5+6 b ;(b \neq 5 a) \\
& 3+2 \cdot 3 b+4=7+6 b ;(b \neq 7 a)
\end{aligned}
$$

If we continue adding even numbers in the equation (1) and (2) we will continue having odd numbers and, between them we will have a great part that they are prime numbers. 


\section{Main results}

Proposition: We analyze the equation (1) and (2) in order that his odd numbers are always prime numbers. For it we must prevent, that equations (1) and (2) they is factorize, therefore $b \neq(5 a ; 7 a)$; of beginning we have $b=[5 a+(1,2,3,4) ; 7 a+(1,2,3,4,5,6)]$ with which the equation (1) and (2) happen to be.

$$
\begin{gathered}
5+6[5 a+(1,2,3,4)] \\
7+6[7 a+(1,2,3,4,5.6)]
\end{gathered}
$$

If now to these equations we divide them respectively by $(5,7)$ and $(7)$ we have.

$$
\begin{gathered}
\frac{5+6[5 a+(1,2,3,4)]}{5,7} \\
\frac{7+6[7 a+(1,2,3,4,5,6)]}{7}
\end{gathered}
$$

with this we reduce the multiples of five or senen.

If we have avoided the multiple of five and seven, the following thing that we will do will be to demonstrate that it is not possible to have numbers that could be a factor. For it we indicate the equations (3) and (4) into arithmetic progressions [2]; it is to say.

$$
\begin{aligned}
& 30 a+11 \\
& 30 a+17 \\
& 30 a+23 \\
& 30 a+29 \\
& 42 a+13 \\
& 42 a+19 \\
& 42 a+25 \\
& 42 a+31 \\
& 42 a+37 \\
& 42 a+43 \\
& 42 a+49
\end{aligned}
$$

In these progressions we have numbers that are factor, this happens when (a) is equal or the multiple one of the odd respective number. To avoid these mistakes, we add in the denominators of the equations ( 3 ) and (4) the respective odd ones of the arithmetic progressions. Therefore

$$
\begin{gathered}
\frac{5+6[5 a+(1 ; 2 ; 3 ; 4)]}{(5 ; 7 ; 11 ; 17 ; 23 ; 29)} \neq Z \\
\frac{7+6[7 a+(1 ; 2 ; 3 ; 4 ; 5 ; 6)]}{(3 ; 5 ; 13 ; 19 ; 31 ; 37 ; 43)} \neq Z
\end{gathered}
$$




\section{Discussion}

We were observing that in the denominator of the second equation previous it appears the number three, this owes to that. If is possible to have in the root of the equation (5) or (6) and odd number that is multiple of a number different from the indicated ones in the denominatot. I demonstrate that the only odd number is the number three, with the following equation.

$$
\begin{gathered}
(2 c+1)(2 b+1)=42 a+(13 ; 19 ; 25 ; 31 ; 37 ; 43 ; 49) \\
4 c b+2 c+2 b=42 a+(12 ; 18 ; 24 ; 30 ; 36 ; 42 ; 48) \\
2 c b+c+b=3(7 a+2) \\
2 c b+c+b=3(7 a+3) \\
2 c b+c+b=3(7 a+4)
\end{gathered}
$$

I demonstrate that does not exist any number, product of two odd ones for the equation (5) with the exception of his denominators, and in turn that the equation (6) admits as value the number three. Therefore having in the denominator of the equation (6) the number three we rejected as possible mistake to say, that the numbers that define the equations do not be a prime number.

Therefore any entire number that does not belong to the equation (5) or (6) is of absolute form a prime number.

Later we define the equation for the twins prime numbers. It is to say that if we take the values of the numerator in the equations (3) and (4) we say that:

$$
7+6[7 a+(1 ; 2 ; 3 ; 4 ; 5 ; 6)]-5+6\left[5 a^{\prime}+(1 ; 2 ; 3 ; 4)\right]=2
$$

And therefore: $[7 a+(1 ; 2 ; 3 ; 4 ; 5 ; 6)]=\left[5 a^{\prime}+(1 ; 2 ; 3 ; 4)\right]$ with these equalities we will define the equation to know the twins prime numbers directly, since:

$$
\begin{gathered}
7 a+1=5 a^{\prime}+(2 ; 3 ; 4) \\
7 a+2=5 a^{\prime}+(1 ; 3 ; 4) \\
7 a+\ldots=5 a^{\prime}+(\ldots \ldots) \\
7 a+. .=5 a^{\prime}+(\ldots) \\
7 a+\ldots=5 a^{\prime}+t(\ldots) \\
7 a+6=5 a^{\prime}+(1 ; 2 ; 3 ; 4)
\end{gathered}
$$

All these equalities diminish to the following ones:

$$
\begin{aligned}
& 7 a \pm 1=5 a^{\prime} \\
& 7 a \pm 2=5 a^{\prime} \\
& 7 a \pm 3=5 a^{\prime} \\
& 7 a+4=5 a^{\prime}
\end{aligned}
$$
$\infty)$

Therefore the equation for any twin prime number is in the following form, with $(p>7) ;(a=0 \rightarrow$

$$
[7+6[7 a \pm(1 ; 2 ; 3 ; 4)]-2 \neq 5 n
$$




\section{Conclusion}

With this article we have solved the four problems that Edmun Landau mentioned unattackable at the present state of science in the mathematicians' fifth International Congress (1912).

-Golbach Conjecture: the sum of two prime numbers, majors that two is always an even number.

$$
\begin{aligned}
& {[5+6(5 a+1]+[5+6(5 a+2)]=2 n} \\
& {[5+6(5 a+1)]+[5+6(5 a+3)]=2 n} \\
& {[\ldots \ldots \ldots \ldots \ldots . . .]+[\ldots \ldots \ldots \ldots \ldots . . .]=2 n}
\end{aligned}
$$

- Conjecture of the twin prime numbers: there are infinite prime numbers $(\mathrm{p})$ such that $(p+2)$ also it is.

$$
\forall([7+6(7 a \pm(1 ; 2 ; 3 ; 4)]-2 \neq 5 n)
$$

; is an twin prime numbers

-Legendre Conjecture: always exist a prime numbers between two perfect squares $n^{2}+m^{2} ; \mathrm{n} ; \mathrm{m}$.

is truth because $n^{2} \pm x=6[5 a+(1 ; 2 ; 3 ; 4)]$ or $n^{2} \pm x=6[7 a+(1 ; 2 ; 3 ; 4 ; 5 ; 6)]$

- The conjecture of that there are infinite prime numbers in the form $\left(n^{2}+1\right)$.

is to say.

$$
\left(\begin{array}{c}
5+6[5 a+(1 ; 2 ; 3 ; 4)] \\
7+6[7 a+(1 ; 2 ; 3 ; 4 ; 5 ; 6)]
\end{array}\right)=n^{2}+1
$$

therefore we have that.

$$
\begin{gathered}
\frac{n^{2}}{2}=2+3[5 a+(1 ; 2 ; 3 ; 4)] \\
\frac{n^{2}}{6}=1+[7 a+(1 ; 2 ; 3 ; 4 ; 5 ; 6)]
\end{gathered}
$$

Finally any prime numbers verify the equations (5) or (6) example.

$(22271 ; 22273)$

$$
7+6[7 \times 5530+1]-2=\mathbf{2 2 2 7 3}-\mathbf{2}=\mathbf{2 2 2 7 1}
$$

example 2

$(1000000000061 ; 1000000000063)$

$$
7+6[7 \times 23809523811-1]-2=\mathbf{1 0 0 0 0 0 0 0 0 0 0 6 3}-\mathbf{2}=\mathbf{1 0 0 0 0 0 0 0 0 0 0 6 1}
$$

example 3

$(33333331 ; 3333331 ; 33331)$

$$
\begin{aligned}
7+6[7 \times 793650+4] & =\mathbf{3 3 3 3 3 3 3 1} \\
7+6[7 \times 79364+6] & =\mathbf{3 3 3 3 3 3 1} \\
7+6[7 \times 793+3] & =\mathbf{3 3 3 3 1}
\end{aligned}
$$

example 4 


\section{REFERENCES}

[1] L.K.Hua, additive theory of prime number, translation of Mathematical monographs, American Mathematical Sociaty vol 13; 1965

[2] Joan Dirichelet, on primes in arithmetic progression $a+b n, 1987$

[3] , a new error analysis for Brun's constant, Virginia Journal of Sciences 52. Springer 2001

[4] R. Crandall and Pomerance, prime numbers a computational perspective, Springer Verlag, New York 2001.

[5] M. C. LIU and T.Z. Wang, on the vinogradov bound in three primes Golbach Conjecture, Acta Arithmetic 105,2002

[6] Bertil Nyman and Thomas R. Bien, new primes gaps betwen 1 e 15 and 5 e 16, Journal of Integer Squences 6, 2003.

[7] Julian Havi, gamma exploring Euler's constant, Princeton University Press, 2003.

[8] B. Cook, A. Magyar and Titichetrakun,a mudimensional Szemerdi theorem in the primes, arxiV: 1306.3025

[9] Daniel Alan Goldston, Yoichi Motohashi, Janes Pintz and Cem Yalcin Yildirim, small gaps betwen primes exist,Poceeding of the Japan Academy, Series A. Methematical Sciences82, 2006

[10] B. Green and T. Tao, the primes contain arbitrarily long arithmetic progression, An. O Math, 167 N2, $481-547$ (2008) 


\section{Erratum}

Abstract: In this annexe I do two properness is done on the article.

\section{Introduction}

In the equation (5) and (6) I modify the denominators. We place in every denominator all the numbers that has both; it is to say.

$$
\begin{gathered}
\frac{5+6[5 a+(1 ; 2 ; 3 ; 4)]}{(3 ; 5 ; 7 ; 11 ; 13 ; 17 ; 19 ; 23 ; 29,31 ; 37 ; 43)} \neq Z \\
\frac{7+6[7 a+(1 ; 2 ; 3 ; 4 ; 5 ; 6)]}{(3 ; 5 ; 11 ; 13 ; 17 ; 19 ; 23 ; 29 ; 31 ; 37 ; 43)} \neq Z
\end{gathered}
$$

The motive of this change is to avoid certain mistakes thet originate when a prime numbers multiples (whose origin is in one of the equations) for one of the numbers of the denominator of other one. Example.

$$
\begin{gathered}
{[5+6[5 \times 2+1]] 31=2201} \\
2201=5+6[5 \times 73+1]
\end{gathered}
$$

not ptime numbers

Therefore the equation for any twin prime number is in the following form, with $(p>7) ;(a=0 \rightarrow$ $\infty)$

In the equation for the prime numbers the sign $( \pm)$ is replaced by the sign $(+)$. I turn I refer to the value that will have always $\left(7 a^{\prime}+(1 ; 2 ; 3 ; 4)\right)$; this value will by exclusively the one that fulfills the equality $\left[\left(7 a^{\prime}+(1 ; 2 ; 3 ; 4)=\right.\right.$ $5 a+(1 ; 2 ; 3 ; 4)]$

$$
\left[7+6\left[7 a^{\prime}+(1 ; 2 ; 3 ; 4)\right]-2 \neq 5 n\right.
$$

Within this annexe we have demonstrated absolutely the crux of the prime numbers. 


\section{Error in the Equations for Prime Numbers of Andri Lopez}

Abstract In this article I present error in the equations for the primes numbers of Andri Lopez. It is absolutely true that all prime number verifies the equations; however it is not true thet all number $(5+6[5 a+(1 ; 2 ; 3 ; 4)]$ or $(7+6[7 a+(1 ; 2 ; 3 ; 4 ; 5 ; 6)]$ thet is not a mukltiple of any of the number $(3 ; 5 ; 7 ; 11 ; 13 ; 17 ; 19 ; 23 ; 29 ; 31 ; 37 ; 43)$ is prime

\section{Main Results}

The error is in the product between the two equations. There are values for $(30 a+(11 ; 17 ; 23 ; 29))$ that are not multiples of any of the numbers of the denominator and we would say that are prime (false). The error originated in a single value for the group of seven; specifically $(7+6[7 b+(1 ; 2 ; 3 ; 4 ; 5 ; 6)])$ with $(b=3)$.

\section{Demonstration}

Throughout the product between the two equations always we have a value .

$$
[(30 a+(11 ; 17 ; 23 ; 29)] \times[(42 b+(13 ; 19 ; 25 ; 31 ; 37 ; 43)]=30 c+(11 ; 17 ; 23 ; 29)
$$

The only term that is not a multiple of six is always the product of two integers (conductores), $(p=(11 ; 17 ; 23 ; 29))$ and $p_{1}=(13 ; 19 ; 31 ; 37 ; 43)$. If now we subtract the value of $(11 ; 17 ; 23 ; 29)$ is become multiples of six and we can divide both terms.

$$
\begin{gathered}
30 a \times 42 b+30 a \times p_{1}+p \times 42+p \times p_{1}=30 c+(p) \\
30 a \times 42 b+30 a \times 11+11 \times 42 b+11 \times 13=30 c+(11 ; 17 ; 23 ; 29) \\
1260 a b+330 a+462 b+143-29=30 c
\end{gathered}
$$

again we have the numbers $(462 ; 143)$ not multiple of five, which prevents us from having a value of an integer in (c). We know that the minor odd number which causes a different odd number from it, is number three.

$$
\begin{gathered}
3 \times(1 ; 2 ; 3 ; 4 ; 6 ; 7 \ldots \ldots \ldots \ldots)+Y=5 n \\
5 \times(1 ; 2 ; 3 ; 4 ; 5 ; 6 ; 7 \ldots \ldots \ldots . .)=5 n
\end{gathered}
$$

Therefore if $(b=(5 ; 5 n)$ not will we have in the sum of the two numbers, a multiple of five; and if we have a multiple of five for a value $(b=3)$.

$$
\begin{gathered}
p \times 42 b+p \times p_{1}-(p)=5 x \\
462 \times 3+143-29=5 x \\
\frac{1380+114}{5}=300
\end{gathered}
$$

therefore the value of $(c)$ is.

$$
\begin{gathered}
c=\frac{1260 a 3+330 a+462 \times 3+114}{30} \\
c=\frac{3780 a+390 a+1386+114}{30} \\
c=137 a+50
\end{gathered}
$$

we observe that the prime number $(137 \notin(7+6[7 \times 3+(1 ; 2 ; ; 3 ; 4 ; 5 ; 6)])$ and as we shall see, is a condition for the error to be fulfilled.

substituting this value $(c)$ in $(30 c+29)$ and have.

$$
30\left[a \times p+\frac{42 p \times 3+p \times p_{1}-(p)}{30}\right]+(p)
$$


This happens in the multiplication process only with $(\mathrm{b}=3)$. Therefore include in the denominator of equations, the values the prime numbers $\mathrm{p}_{3}=(139 ; 151 ; 157 ; 163)$, and have now the problem solved.

The error exists in: $\forall\left(Z_{p}\right) \geq 41$.

$\left(\mathrm{Z}_{p}\right) \times p_{3}=30 \mathrm{c}+(\mathrm{p})$

example, $\left(\mathrm{Z}_{p}\right) \neq 43$ :

$$
\begin{gathered}
41 \times 139=5699 \\
5+6[5 \times 189+4]=5699
\end{gathered}
$$

Therefore the equations for all prime numbers are in the form:

$$
\frac{5+6[5 a+(1 ; 2 ; 3 ; 4)]}{3 ; 5 ; 7 ; 11 ; 13 ; 17 ; 19 ; 23 ; 29 ; 31 ; 37 ; 43 ; 139 ; 151 ; 157 ; 163} \neq Z
$$

is prime.

$$
\frac{7+6[7 a+(1 ; 2 ; 3 ; 4 ; 5 ; 6)]}{3 ; 5 ; 7 ; 13 ; 19 ; 31 ; 37 ; 43} \neq Z
$$

is prime

\section{REFERENCES}

[1] equation for all prime numbers. Universal Journal of Computational Mathematics Vol 1 (3); 2013. 


\section{Recent Errors in the Equations of Andri Lopez}

They are in the product of prime numbers of the seven gtoup and in the power of prime numbers.

(i) The last error we have is in the product two primes of the group seven; is solved with the following equations.

$$
\begin{gathered}
\frac{N-13[19 ; 37 ; 43]}{42} \neq Z \\
\frac{N-[19(31 ; 37 ; 43)]}{42} \neq Z \\
\frac{N-31[37 ; 43]}{42} \neq Z \\
\frac{N-[37 \times 43]}{42} \neq Z
\end{gathered}
$$

Example:

$$
\begin{gathered}
79 \times 127=10033 \\
10033=7+6[7 \times 238+5] \\
\frac{10033-37 \times 43}{42}=201
\end{gathered}
$$

(ii) There is also a error in the equations for $\left(p^{n}=N\right)$.

$$
\forall\left(p^{n}\right) \rightarrow 30 a^{\prime}+(p)=42 a+\left(p_{1}\right)
$$

example:

$$
\begin{gathered}
1889423440969 \\
\frac{1889423440969-43}{42}=44986272403 \\
(1374563)^{2}=1889423440969
\end{gathered}
$$

To avoid this error we have the following equation.

$$
\begin{gathered}
\frac{N-(19 ; 43)}{(30 ; 42)} \neq Z \\
\frac{1889423440969-[19 ; 43]}{(30 ; 42)}=\begin{array}{l}
62980781365 \\
44986272403
\end{array}
\end{gathered}
$$

\section{Conclusion}

All known prime number such as; Dirichelit numbers prome, Euler,Mersen, Fermat fulfill the equation of origin $(5+6[5 a+(1 ; 2 ; 3 ; 4)])$ or $(7+6[7 a+(1 ; 2 ; 3 ; 4 ; 5 ; 6)])$. In this work I demonstrated, because, that all the (a) not have a prime number. therefore. A number is prime if and only If, comply the following equations.

$$
\begin{gathered}
\frac{N}{(3 ; 5 ; 7 ; 11 ; 13 ; 17 ; 23 ; 29 ; 31 ; 37 ; 43 ; 139 ; 151 ; 157 ; 163)} \neq Z \\
\frac{N-(19 ; 43)}{(30 ; 42)} \neq Z
\end{gathered}
$$




$$
\begin{gathered}
\frac{N-13[19 ; 37 ; 43]}{42} \neq Z \\
\frac{N-[19(31 ; 37 ; 43)]}{42} \neq Z \\
\frac{N-31[37 ; 43]}{42} \neq Z \\
\frac{N-[37 \times 43]}{42} \neq Z
\end{gathered}
$$

The distribution of prime numbers is always in every interval.

between: $(30 a+11)$ and $(42 a+43)$. The number of primes per interval ranges from a minimum of two and a maximum of seven.

\section{REFERENCES}

[1] equation for all prime numbers. Universal Journal of Computational Mathematics Vol 1 (3); 2013. 